\title{
Special issue with selected papers from 2017 Brazilian Symposium on Computer Engineering (SBESC 2017)
}

\author{
José Augusto Miranda Nacif ${ }^{1}$ - Marcio Seiji Oyamada² \\ Published online: 22 April 2019 \\ (c) Springer Science+Business Media, LLC, part of Springer Nature 2019
}

In 2017, the Embedded Systems track of the Brazilian Symposium on Computing Systems Engineering (SBESC) received many high quality submissions. According to the ranking produced by the Program Committee, authors of four top submissions have been invited to submit revised and extended versions of their papers for consideration by this journal. After a strict review process we selected three articles for inclusion in this special issue.

The first paper is entitled "A Fast and Accurate Hybrid Fault Injection Platform for Transient and Permanent Faults" by Anderson Luiz Sartor, Pedro Henrique Exenberger Becker and Antonio Carlos Schneider Beck. The authors present a hybrid fault injection framework that automatically switches between RTL and gate-level simulation modes, speeding up the simulation time while maintaining gate-level accuracy and controllability.

The second paper is "A distributed algorithm to schedule TSCH links under the SINR model" by José Carlos da Silva and Flávio Assis. The authors propose a distributed algorithm to assign a time slot and a channel to each pair of communicating nodes in industrial wireless sensor networks. This algorithm also takes into consideration interference and has been validated under the Signal-to-Interference-plus-Noise-Ratio (SINR) model.

The third paper is "Trace-driven extension for Noxim simulator" by Ivan Luiz Pedroso Pires, Marco Antonio Zanata Alves and Luiz Carlos Pessoa Albini. The authors present an extension to Noxim simulator for NoC systems, enabling the use of external traces based on messages. The extension includes also the ability to consider the Processing Elements (PEs) processing time as well as the support to Message Passing Interface (MPI) communication on Noxim simulator.

We would like express our gratitude to the Program Committee of SBESC for their hard work on reviewing the papers for the conference, the additional reviewers who helped us with the review process for this special issue, as well as the authors themselves for their excellent contributions.

Guest Editors

José Augusto Miranda Nacif

jnacif@ufv.br

Marcio Seiji Oyamada

marcio.oyamada@unioeste.br

1 Science and Technology Institute, Federal University of Viçosa (UFV), Florestal, MG, Brazil

2 Computer Science Department, Western State University of Parana (UNIOESTE), Cascavel, PR, Brazil 
Publisher's Note Springer Nature remains neutral with regard to jurisdictional claims in published maps and institutional affiliations. 